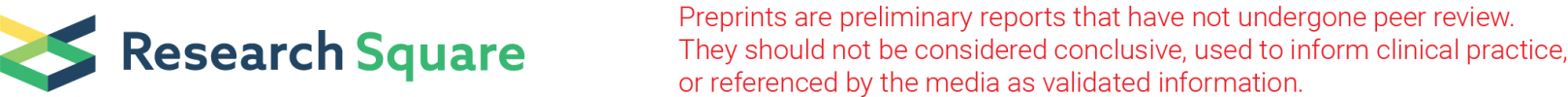

\section{Examining Long-Term Effects of Human Papillomavirus Vaccine Recommendation Messages: A 4-Month Follow-up Survey of a Randomized Controlled Study in Japan}

\section{Tsuyoshi Okuhara ( $\nabla$ okuhara-ctr@umin.ac.jp )}

Tokyo Daigaku Daigakuin Igakukei Kenkyuka Igakubu

Hirono Ishikawa

Teikyo Daigaku

Haruka Ueno

Tokyo Daigaku Daigakuin Igakukei Kenkyuka Igakubu

Hiroko Okada

Tokyo Daigaku Daigakuin Igakukei Kenkyuka Igakubu

Takahiro Kiuchi

Tokyo Daigaku Daigakuin Igakukei Kenkyuka Igakubu

\section{Research article}

Keywords: human papillomavirus (HPV) vaccination, anti-vaccination movement, narrative, persuasion, long-term effect, health communication

Posted Date: June 1st, 2020

DOI: https://doi.org/10.21203/rs.3.rs-31332/v1

License: (c) This work is licensed under a Creative Commons Attribution 4.0 International License.

Read Full License 


\section{Abstract}

Background: We previously conducted a randomized controlled study to examine persuasive messages recommending HPV vaccination to mothers with daughters in September 2017 in Japan. Our previous study showed that the three types of intervention message used (statistical information only, a patient's narrative in addition to statistical information, and a mother's narrative in addition to statistical information) all significantly improved mothers' intention to have their daughter(s) receive the HPV vaccine, in comparison with mothers who received no messaging. The present study is a follow-up survey to assess the long-term effect of the intervention in our previous study.

Methods: Four months after the initial study, in January 2018, participants in the previous study were contacted and queried about their current intention to have their daughter(s) receive the HPV vaccine. Statistical analysis was conducted using the paired t-test and analysis of variance.

Results: A total of 978 mothers participated in the current survey. Vaccination intention 4 months after intervention had decreased to a level that did not differ significantly from the level prior to intervention in all three intervention conditions. The amount of change in vaccination intention 4 months after intervention did not differ significantly among the three intervention groups $(p=0.871)$.

Conclusions: A single exposure to messaging was insufficient to produce a persistent intervention effect; repeated exposure to messaging coupled with other interventions may be important.

\section{Background}

The human papillomavirus (HPV) vaccines have contributed to reduce the public health burden of cervical cancer [1]. However, widespread uptake of the vaccine is necessary for this advance. The situation is critical in Japan. Proactive recommendation of HPV vaccination was suspended by the Ministry of Health, Labour and Welfare in June 2013 in Japan, because of negative campaigns by mass media about severe adverse reactions allegedly caused by HPV vaccination. Since then, the HPV vaccination rate among age-eligible girls has been stagnating, with only 0.3 percent of girls being vaccinated [2]. Individuals have fears concerning adverse reactions to HPV vaccination in Japan and other countries [4-8], although studies have demonstrated the safety of HPV vaccines [1,3]. To sway this biased anti-HPV vaccination sentiment in this critical situation, influential communication tactics are needed to encourage the public to make less biased decisions.

Therefore, we previously conducted a randomized controlled study to examine the types of persuasive messages to use when recommending HPV vaccination to mothers with daughters in September 2017 in Japan [9]. We compared message persuasiveness according to four conditions: messages including statistical information only; statistical messages plus narrative messages of a patient who experienced cervical cancer; statistical messages plus narrative messages of a mother whose daughter experienced cervical cancer; and a control condition [9]. Our previous study showed that messages containing statistics information only, as well as a narrative (of a patient or a mother) in addition to statistical 
messages, significantly increased participants' attitude and intention to have their daughter(s) receive the HPV vaccine, compared with prior to the intervention and with participants who received no messaging $(p<0.001)$ [9]. This result indicated that exposure to messaging about efficacy and safety of HPV vaccine may increase vaccination intention of mothers with daughters directly after exposure to messaging, whether this includes only statistical messages, or narratives of experiences with cervical cancer in addition to statistical messages. Additionally, our previous study showed that the intention for the statistical messages plus mother's narrative was the highest among the intervention groups and was significantly higher than in the statistics-only group $(p=0.040)$ [9]. This indicated that the narrative messages of a mother whose daughter experienced cervical cancer may be persuasive for audiences who are mothers with daughters.

Our previous study only assessed participants' vaccination intention directly after exposure to messaging. The long-term effects of messages should be investigated because HPV vaccination expects multiple injections given over a series of weeks: in Japan, the second dose is given 1 month after and the third dose is given 6 months after the first dose. One previous study examined the impact of an individually tailored intervention on knowledge, risk perception, intention to be vaccinated, and uptake regarding HPV vaccine, among unvaccinated female university students over 3 months following the intervention [10]. However, no studies have examined the long-term effects of intervention among parents of adolescent daughters. Therefore, in the present study, we contacted participants in our previous study 4 months after the initial investigation and reassessed their intention to have their daughter(s) receive the HPV vaccine. The present study aimed to examine whether the effects of intervention persisted 4 months after the intervention, and whether the degree of persistence of the intervention effect depends on the type of message. We set the following research questions.

Research question 1: Does vaccination intention 4 months after intervention differ significantly from the intention before intervention?

Research question 2: Does the amount of change in vaccination intention 4 months after intervention differ significantly among the three types of message?

\section{Methods}

\section{Summary of the previous study}

Detailed information was reported in our previous study [9]. Briefly, in that study, participants were recruited from among individuals registered in a survey company database in Japan. Eligibility criteria were mothers with a daughter(s) aged 12-16 years who had never received HPV vaccination. A total of 1432 mothers completed our previous web-based survey in September 2017. Participants were randomly assigned to a group that received statistical messages only $(n=394)$, a group that received a message that included statistical information plus a patient's narrative $(n=408)$, a group that received statistical information plus a message including a mother's narrative $(n=411)$, or a control group that received no message $(n=219)$, using an algorithm included in the survey computer program. All participants were 
asked sociodemographic information, history of cancer and sexually transmitted diseases, and whether they knew about the media coverage regarding adverse reactions to the HPV vaccine and suspension of the proactive recommendation for HPV vaccination by the Japanese government. Regarding the outcome, intention to have one's daughter(s) receive the HPV vaccine was assessed before and directly after intervention.

Regarding the intervention materials, the statistical information conveyed morbidity and mortality of cervical cancer and efficacy and safety of HPV vaccine. The content was identical in the intervention materials presented to the three groups. For the narrative content, the narrator recounted their experience of being diagnosed with cervical cancer, giving up having children, developing from complications, fearing cancer recurrence, and recommending HPV vaccination. The narrative contents were identical for the intervention materials presented to the groups receiving a patient's and mother's narratives, except the subject of the narrative differed (i.e., "I" in the patient's narrative and "my daughter" in the mother's narrative). The statistical message and mother's narrative used in our previous study is provided in the Appendix, which was translated into English for this report.

\section{Methods of the present study}

Four months after the initial study, we conducted a follow-up survey. Participants in our previous study were contacted by e-mail and recruited to participate in the follow-up survey in January 2018. Those who consented to participate completed this follow-up survey online. Participants were asked about their intention to have their daughter(s) receive the HPV vaccine. In other words, participants reported their vaccination intention before and directly after the intervention in our previous study, as well as 4 months after the intervention in the present study. As in our past study, participants' intention to vaccinate was measured according to their responses to the following three questions: (1) "How likely are you to have your daughter(s) receive the HPV vaccine sometime soon?"; (2) "If you were faced with the decision of whether to have your daughter(s) receive the HPV vaccine today, how likely is it that you would choose to have her receive the vaccine?"; and (3) "How likely is it that you would have your daughter(s) receive the HPV vaccine in the future?". The following responses were given 1 to 6 points, respectively: "very unlikely", "unlikely", "somewhat unlikely", "somewhat likely", "likely", and "very likely". A mean score was used in the analysis. Higher scores indicated greater intention to vaccinate. This measure was adapted from a previous publication [11].

Descriptive statistics were used to summarize participants' sociodemographic information and history, using percentage for categorical variables and mean \pm standard deviation (SD) for continuous variables. Those variables were compared among groups using the chi-square test, Fisher's exact test, and analysis of variance (ANOVA). Participants' vaccination intention was compared between before and 4 months after intervention, using the paired $t$-test. When no variable differed significantly among groups, ANOVA was conducted with changes in the intention to vaccinate before and 4 months after intervention as the dependent variable and the group assignment as the independent variable. Tukey's test was conducted on the significant main effects, where appropriate. The Games-Howell post-hoc test was performed 
when the assumption of homogeneity of variances was not satisfied. When there was a variable that significantly differed among groups, analysis of covariance (ANCOVA) was conducted with changes in the intention to vaccinate before and 4 months after intervention as the dependent variable, the group assignment as the independent variable, and the variable that significantly differed among groups as the covariate. Bonferroni correction was applied for the post hoc test to compare adjusted means of intentions. A $p$-value of $<0.05$ was set as significant in all statistical tests. All statistical analyses were performed using IBM SPSS Statistics for Windows, Version 21.0 (IBM Corp., Armonk, NY, USA).

The protocol was approved by the ethical review committee of the Graduate School of Medicine, The University of Tokyo. All participants gave their written informed consent in accordance with the Declaration of Helsinki.

\section{Results}

\section{Participant characteristics}

A total of 978 mothers participated in this follow-up survey; the response rate was $57 \%$. A total 258 participants were included in the group that received statistical messages only, 269 were in the group that received a patient's narrative plus statistical messages, and 284 were in the group that received a mother's narrative plus statistical messages, and 167 in the control group. Table 1 shows the participants' characteristics. Participant age ranged from 31 to 61 years (mean 44 years, SD 4.5). A total $52 \%$ of participants' daughters were 12-14 years old and the remainder were $15-16$ years old. About $90 \%$ of participants had not been advised by health professionals that their daughter(s) should receive the HPV vaccine, and knew about the media coverage regarding adverse reactions to the HPV vaccine and suspension of the proactive recommendation for HPV vaccination by the Japanese government. About $90 \%$ of participants did not have a history of cervical cancer or sexually transmitted diseases. Participants' characteristics were not significantly different among groups.

\section{Intervention effect}

The internal consistency of questions regarding the intention to have one's daughter(s) receive the HPV vaccine was excellent (Cronbach's $\mathrm{a}=0.947$ ). The mean scores for participants' intention to have their daughter(s) receive the HPV vaccine before, directly after, and 4 months after intervention in the three intervention groups were 2.51 (SD 0.95), 2.82 (SD 0.99), and 2.59 (SD 0.97), respectively. The mean of intention in the control group was 2.28 (SD 0.91) in our previous study, and 2.36 (SD 0.92) in the present study. Figure 1 shows the mean vaccination intention at baseline, directly after, and 4 months after intervention across the groups. The mean vaccination intention decreased from directly after intervention to 4 months after intervention: 2.82 (SD 1.00) to 2.62 (SD 1.00) in the statistics only group; 2.84 (SD 1.01) to 2.60 (SD 0.97) in the statistics plus patient's narrative group; and 2.78 (SD 0.96) to 2.54 (SD 0.95) in the statistics plus mother's narrative group. Table 2 shows changes in the intention to vaccinate before intervention and 4 months after intervention in the three intervention groups. The paired $t$-test revealed that vaccination intention 4 months after intervention did not differ significantly from the intention before 
intervention in the intervention groups ( $p=0.256$ in the statistics-only group, $p=0.054$ in the statistics plus patient's narrative group, $p=0.096$ in the statistics plus mother's narrative group). Also, in the control group, vaccination intention 4 months after intervention did not differ significantly from the intention before intervention $(p=0.147)$. ANOVA revealed no main effect of group assignment on the amount of change in vaccination intention 4 months after intervention $[F(2,808) 0.138, p=0.871]$; this indicated that the amount of change in vaccination intention 4 months after intervention did not significantly differ among the three intervention groups.

\section{Discussion}

We examined whether the effect of an intervention recommending HPV vaccination for mothers with daughters had persisted 4 months after the intervention, and whether the degree of persistence of the intervention effect depended on the type of message: a statistical message only, a patient's narrative in addition to statistical messages, or a mother's narrative in addition to statistical messages.

The results of the present study showed that vaccination intention in the all groups slightly increased, although those changes were not significant. The effect of intervention had not persisted at 4 months after intervention, and the degree of persistence of the intervention effect 4 months after intervention did not differ among the three message types. More precisely, vaccination intention 4 months after intervention decreased to a level that did not significantly differ from the level before intervention under all three intervention conditions. Thus, the answer to our research question 1 was "no". However, in the group that received a patient's narrative plus statistical messages, the difference of vaccination intention between before and 4 months after intervention was marginally significant. The number of participants in the present study was smaller than that in our previous study because of loss to follow-up. We conducted post-hoc power analyses; the statistical power in the patient's narrative plus statistical messages condition was $55 \%$. The analysis may have been underpowered to detect the modest difference that would be expected if there were an appropriate sample size.

The amount of change in vaccination intention 4 months after intervention did not differ significantly among the three message types. Thus, the answer to our research question 2 was also "no". Our previous study showed that vaccination intention increased significantly directly after reading intervention materials, and that statistical messages plus a mother's narrative increased vaccination intention the most [9]. However, the present findings revealed that those effects wore off during the 4 months following intervention. Our results are consistent with those of a previous study showing that risk perception, intention to be vaccinated, and HPV vaccine uptake did not differ significantly between baseline and 3 months following intervention among female university students in the United States [10].

HPV vaccination expects multiple injections given over a series of weeks. In Japan, the second dose is given 1 month after the first dose, and the third dose is given 6 months after the first dose. Studies indicate that there are a number of anti-HPV vaccination websites on the internet [12] and that seeing anti-vaccination messages online can negatively affect the audience's attitude toward vaccination, even 5 
months after exposure to these messages [13]. Considering this, persistently increased vaccination intention after an intervention to promote HPV vaccines is important, to effectively conduct vaccination.

To maintain the intervention effect, intervention messages should be easy to remember so that they can be recalled over time by the message recipients [14]. Studies indicate that narratives are more easily remembered than didactic content, and narratives are the preferred mental structure for storing and retrieving information $[15,16]$. Events and characters in narrative messages are linked to each other through personal, causal, temporal, and spatial associations, which facilitate storage and retrieval of more complex information because the recipient need only remember a single story rather than miscellaneous information [17]. Studies indicate that health materials with narrative messages enhance recall and are associated with lower decision conflict than messages without narratives [18-20]. However, the advantage of narratives for memory and recall did not seem to be present in this study, considering that the intervention effect of narratives in addition to statistical messages diminished over time to the same degree as the effect of statistical messages only. The reason may be that the narratives used in the present study were created for research purposes and thus may have been too short and uninteresting to be remembered and recalled over time by participants. It may be useful to examine the persuasiveness of a longer and more vivid narrative of an experience of cervical cancer in future studies, as the narrative of a patient or a mother is more likely to be retained and recalled and will consequently influence decision making regarding vaccination over time.

A review of studies about media exposure indicate that the degree of exposure to health messaging among message recipients is positively associated with the likelihood of their engagement in health behaviors [21]. It is considered that repeated exposure to health messages enhances recognition and recall of messages among recipients and encourages them to undertake certain health behaviors [22]. To improve persistence of the intervention effect of messages recommending HPV vaccination, it may be important for health institutes to frequently disseminate messages such that target populations are repeatedly exposed to those messages. However, the mean vaccination intention directly after intervention was less than 3 (i.e., somewhat unlikely) in our previous study [9]. Frequent messaging alone may be insufficient to increase vaccine uptake. Messaging coupled with other interventions, such as facilitating access to the vaccine and providing educational programs, may help to improve vaccine uptake.

Additional studies indicate that educational programs on topics such as anti-stigma toward mental illness and self-management of chronic illness have improved participant's knowledge, attitude, and practices over time [23-25]. Although no studies have examined the effect of educational programs in the context of HPV vaccination as far as we know, provision of educational programs that communicate the benefits and risks of HPV vaccines in a balanced manner may contribute to persistently increased vaccination intention among participants. Especially in Japan where negative attitudes toward HPV vaccines focusing on the risk of vaccination have been established, educational programs that provide a set of facts and narratives and clarify misunderstandings regarding HPV vaccines may be more influential for participants' long-term decision making than only disseminating written messages. 
Several limitation should be considered in this study. First, when the persuasive intent is obvious, narrative persuasion is hindered because some audiences may resist if they feel they are being manipulated [26]. This constraint may be related to the negative results of the present study and should be noted, in addition to the brief content of the materials, as discussed above. Second, we did not examine the duration of the intervention effect, i.e., how many weeks did the intervention effect persist? Investigating this issue is necessary so as to determine the appropriate frequency of message exposure to sustain increased vaccination intention. Third, we assessed vaccination intention rather than vaccine uptake. However, behavioral intention is generally measured in public health studies because it predicts an actual behavior [27]. Fourth, whether some participants had their daughter(s) receive HPV vaccines after intervention is unknown. However, the influence of this on the study results is considered to be small because the HPV vaccination rate is only a few percent in Japan. Fifth, factors other than intervention, such as exposure to media coverage, may have influenced vaccination intention among some participants during the 4 months after intervention. Sixth, the response rate in the present study was 57\%; the intention of the $43 \%$ of participants who did not respond to the follow-up survey are unknown. The respondents in the present study could be self-selected in the follow-up survey. Selection bias may have influenced the study results. Finally, the sample size in this follow-up study was smaller than that in our initial study. The analyses in the present study may have been underpowered to detect the differences that would be expected if there were appropriate sample sizes.

\section{Conclusions}

Our previous study showed that HPV vaccination intention increased significantly directly after intervention using statistical messages only, a patient's narrative in addition to statistical messages, and a mother's narrative in addition to statistical messages. However, the present study showed that the effect of intervention wore off during the 4 months following intervention, and that the degree of persistence of the intervention effect 4 months after intervention did not differ among the three message types. It is important that increased vaccination intention is maintained because HPV vaccination expects multiple injections given over a series of weeks. To maintain the intention of HPV vaccination, a single message exposure may be insufficient.

Messaging coupled with other interventions, such as facilitating access to the vaccine and providing educational programs, may be more effective. These types of approaches should be examined in future studies.

\section{Abbreviations}

SD: standard deviation

HPV: human papillomavirus

ANOVA: analysis of variance 
ANCOVA: analysis of covariance

\section{Declarations}

\section{Ethics approval and consent to participate}

The protocol was approved by the ethical review committee at the Graduate School of Medicine, University of

Tokyo (number 11624). This study was carried out in accordance with the recommendations of the ethical review committee at the Graduate School of Medicine, The University of Tokyo.

All participants gave written informed consent in accordance with the Declaration of Helsinki.

\section{Consent for publication}

Not applicable

\section{Availability of data and materials}

The datasets used and/or analysed during the current study are available from the corresponding author on reasonable request.

\section{Competing interests}

The authors declare that they have no competing interests.

\section{Funding}

This study was supported by JSPS KAKENHI (grant number 16K01752, 19K10615). The funding body has no role in the design of the study and collection, analysis, and interpretation of data and in writing the manuscript.

\section{Authors' contributions}

TO conceived the concept of the study, collected data, analyzed data. TO, HI, HU, HO and TK discussed and interpreted the results. TO wrote the manuscript. HI provided the first feedback for the manuscript. TO, HI, HU, HO and TK discussed the manuscript critically and provided feedback for the manuscript. TO revised the manuscript. TO, HI, HU, $\mathrm{HO}$ and TK approved the final version of the manuscript.

\section{Acknowledgements}

We thank Edanz Group (www.edanzediting.com/ac) for editing a draft of this manuscript.

\section{References}


1. Meeting of the Global Advisory Committee on Vaccine Safety, 7-8 June 2017. Wkly Epidemiol Rec;2017: 92:393-402. http://apps.who.int/iris/bitstream/10665/255870/1/WER9228.pdf?ua=1

2. The Ministry of Health, Labour and Welfare. Vaccine coverage. 2018. https://www.mhlw.go.jp/topics/bcg/other/5.html

3. Iwata S, Okada K, Kawana K. Consensus statement from 17 relevant Japanese academic societies on the promotion of the human papillomavirus vaccine. Vaccine. 2017;35:2291-2292. doi: 10.1016/j.vaccine.2017.03.015

4. Larson $\mathrm{H}$, Wilson R, Hanley S, Parys A, Paterson P. Tracking the global spread of vaccine sentiments: the global response to Japan's suspension of its HPV vaccine recommendation. Hum Vaccin Immunother. 2014;10:2543-2550. doi: 10.4161/21645515.2014.969618

5. Dornbusch H, Stiris T, Del Torso S, Ross-Russell R, Zavrsnik J, Wettergren B, et al. Human papillomavirus vaccination crisis in Japan. J Paediatr Child Health. 2015;51:1146-1147. doi: $10.1111 /$ jpc. 13020

6. Darden PM, Thompson DM, Roberts JR, Hale JJ, Pope C, Naifeh M, et al. Reasons for not vaccinating adolescents: National immunization survey of teens, 2008-2010. Pediatrics. 2013;131:645-651. doi: 10.1542/peds.2012-2384

7. Wong M, Lee A, Ngai K, Chor J, Chan P. Knowledge, Attitude, practice and barriers on vaccination against human papillomavirus infection: A cross-sectional study among primary care physicians in Hong Kong. Plos ONE. 2013;8:e71827. doi: 10.1371/journal.pone.0071827

8. Sotiriadis A, Dagklis T, Siamanta V, Chatzigeorgiou K, Agorastos T. Increasing fear of adverse effects drops intention to vaccinate after the introduction of prophylactic HPV vaccine. Arch Gynecol Obstet. 2012;285:1719-1724. doi: 10.1007/s00404-011-2208-z

9. Okuhara T, Ishikawa H, Okada M, Kato M and Kiuchi T. Persuasiveness of Statistics and Patients' and Mothers' Narratives in Human Papillomavirus Vaccine Recommendation Messages: A Randomized Controlled Study in Japan. Front Public Health. 2018;6:105. doi: 10.3389/fpubh.2018.00105

10. Bennett AT, Patel DA, Carlos RC, Zochowski MK, Pennewell SM, Chi AM, Dalton VK. Human papillomavirus vaccine uptake after a tailored, online educational intervention for female university students: a randomized controlled trial. Journal of Women's Health. 2015;24: 950-957.

11. Nan X, Madden K. HPV vaccine information in the blogosphere: How positive and negative blogs influence vaccine-related risk perceptions, attitudes, and behavioral intentions. Health Commun. 2012;27:829-836. doi: 10.1080/10410236.2012.661348

12. Okuhara T, Ishikawa H, Okada M, Kato M, Kiuchi T. Contents of Japanese pro- and anti-HPV vaccination websites: A text mining analysis. Patient Educ Couns. 2018;101: 406-413. doi: 10.1016/j.pec.2017.09.014.

13. Betsch, C. Innovations in communication: the Internet and the psychology of vaccination decisions. Euro Surveill. 2011;16:1-6. doi: 10.2807/ese.16.17.19849-en 
14. McGuire WJ. "McGuire's classic input-output framework for constructing persuasive messages". In: Rice RE, Atkin CK, editors. Public communication campaigns, 4th edition. Thousand Oaks, CA: SAGE Publications. 2013. p. 133-46.

15. Schank RC, Abelson RP. Knowledge and memory: The real story. In: Wyer RJ, editor. Advances in social cognition. Vol. 8. Hillsdale, NJ: Lawrence Erlbaum Associates. 1995. p. 1-85.

16. Schank RC, Berman TR. The pervasive role of stories in knowledge and action. In: Green MC, Strange JJ, Brock TC, editors. Narrative impact: Social and cognitive foundations. Mahwah, NJ: Lawrence Erlbaum Associates. 2002. p. 287-313.

17. Green MC, Brock TC. Persuasiveness of narratives. In: Brock TC, Green MC, editors. Persuasion: Psychological insights and perspectives, 2nd edition. Thousand Oaks, CA: Sage Publications. 2005. p. 117-42.

18. Kreuter MW, Holmes K, Alcaraz K, Kalesan B, Rath S, Richert M, et al. Comparing narrative and informational videos to increase mammography in low-income African American women. Patient Educ Couns. 2010;81:6-14. doi:10.1016/j.pec.2010.09.008

19. Volk RJ, Jibaja-Weiss ML, Hawley ST, Kneuper S, Spann SJ, Miles BJ, Hyman DJ. Entertainment education for prostate cancer screening: a randomized trial among primary care patients with low health literacy. Patient Educ Couns. 2008;73:482-9. doi: 10.1016/j.pec.2008.07.033

20. Dillard A, Fagerlin A, Dal Cin S, Zikmund-Fisher B, Ubel P. Narratives that address affective forecasting errors reduce perceived barriers to colorectal cancer screening. Soc Sci Med. 2010;71:45-52. doi:10.1016/j.socscimed.2010.02.038

21. Morris DS, Rooney MP, Wray RJ, Kreuter MW. (2009). Measuring exposure to health messages in community-based intervention studies: a systematic review of current practices. Health Educ Behav. 2009;36:979-998. doi: 10.1177/1090198108330001

22. O'Hara BJ, Bauman AE, Phongsavan P. Using mass-media communications to increase population usage of Australia's Get Healthy Information and Coaching Service ${ }^{\circledR}$. BMC Public Health. 2012;12:762. doi: 10.1186/1471-2458-12-762

23. Niedermann K, Fransen J, Knols R, Uebelhart D. Gap between short-and long-term effects of patient education in rheumatoid arthritis patients: A systematic review. Arthritis Care Res. 2004;51:388-398. doi: 10.1002/art.20399

24. Thongsai, S., \& Youjaiyen, M. The long-term impact of education on diabetes for older people: a systematic review. Glob J Health Sci. 2013;5:30. doi: 10.5539/gjhs.v5n6p30

25. Corrigan, P., Michaels, P. J., \& Morris, S. Do the effects of antistigma programs persist over time? Findings from a meta-analysis. Psychiatr Serv. 2015;66: 543-546. doi: 10.1176/appi.ps. 201400291

26. Moyer-Guse E, Nabi R. Explaining the effects of narrative in an entertainment television program: Overcoming resistance to persuasion. Hum Commun Res. 2010;36:26-52. doi: 10.1111/j.14682958.2009.01367.x

27. Sheeran P. Intention-behavior relations: A conceptual and empirical review. Eur Rev Soc Psychol. 2002;12:1-36. doi: 10.10 


\section{Tables}

Table 1. Participant sociodemographic information and history

\begin{tabular}{|c|c|c|c|c|c|c|}
\hline & $\begin{array}{l}\text { Total } \\
(\mathrm{n}=978)\end{array}$ & $\begin{array}{l}\text { Statistics } \\
\text { only } \\
(n=258)\end{array}$ & $\begin{array}{l}\text { Statistics and } \\
\text { patient's } \\
\text { narrative } \\
(\mathrm{n}=269)\end{array}$ & $\begin{array}{l}\text { Statistics and } \\
\text { mother's } \\
\text { narrative } \\
(\mathrm{n}=284)\end{array}$ & $\begin{array}{l}\text { Control } \\
(\mathrm{n}=167)\end{array}$ & $p$ \\
\hline Age, mean year (SD) & $\begin{array}{l}43.9 \\
(4.5)\end{array}$ & $43.9(4.5)$ & $43.5(4.3)$ & $44.2(4.9)$ & $\begin{array}{l}43.8 \\
(4.3)\end{array}$ & $0.429^{b}$ \\
\hline \multicolumn{7}{|l|}{ Age of daughters, \% } \\
\hline 12-14 years old & 51.5 & 57.0 & 49.8 & 49.1 & 49.7 & $0.234^{c}$ \\
\hline $15-16$ years old & 48.5 & 43.0 & 50.2 & 50.9 & 50.3 & \\
\hline \multicolumn{7}{|l|}{ Highest education, \% } \\
\hline Less than high school & 3.1 & 2.7 & 2.6 & 4.6 & 1.8 & $0.583^{d}$ \\
\hline High school graduate & 29.7 & 30.6 & 29.7 & 27.7 & 31.7 & \\
\hline Some college & 42.4 & 39.5 & 40.1 & 44.2 & 47.3 & \\
\hline College graduate & 23.8 & 25.6 & 26.4 & 22.8 & 18.6 & \\
\hline Graduate school & 1.0 & 1.6 & 1.1 & 0.7 & 0.6 & \\
\hline \multicolumn{7}{|l|}{ Household income, \% } \\
\hline Less than 2 million yen ${ }^{a}$ & 7.9 & 7.0 & 7.4 & 8.8 & 8.4 & $0.917^{c}$ \\
\hline 2-6 million yen ${ }^{a}$ & 35.5 & 32.9 & 36.4 & 37.2 & 35.3 & \\
\hline More than 6 million yen ${ }^{a}$ & 43.4 & 47.7 & 42.4 & 40.0 & 44.3 & \\
\hline Unknown & 13.2 & 12.4 & 13.8 & 14.0 & 12.0 & \\
\hline \multicolumn{7}{|c|}{$\begin{array}{l}\text { Advised by health professionals to have their daughter(s) } \\
\text { receive HPV vaccines, \% }\end{array}$} \\
\hline Yes & 6.1 & 8.9 & 5.2 & 6.0 & 3.6 & $0.124^{c}$ \\
\hline No & 93.9 & 91.1 & 94.8 & 94.0 & 96.4 & \\
\hline \multicolumn{7}{|c|}{$\begin{array}{l}\text { Knew about media coverage of adverse reactions to HPV } \\
\text { vaccines, \% }\end{array}$} \\
\hline Yes & 90.1 & 87.2 & 90.7 & 89.5 & 94.6 & $0.091^{\mathrm{c}}$ \\
\hline No & 9.9 & 12.8 & 9.3 & 10.5 & 5.4 & \\
\hline \multicolumn{7}{|c|}{$\begin{array}{l}\text { Knew about suspension of the proactive recommendation } \\
\text { for HPV vaccination by the government, \% }\end{array}$} \\
\hline Yes & 86.2 & 83.7 & 87.4 & 85.6 & 89.2 & $0.391^{\mathrm{c}}$ \\
\hline No & 13.8 & 16.3 & 12.6 & 14.4 & 10.8 & \\
\hline \multicolumn{7}{|c|}{ History of cervical cancer including familiar persons, \% } \\
\hline Yes & 8.3 & 7.4 & 7.8 & 9.5 & 8.4 & $0.629^{d}$ \\
\hline No & 91.2 & 92.2 & 91.1 & 90.5 & 91.0 & \\
\hline No answer & 0.5 & 0.4 & 1.1 & 0 & 0.6 & \\
\hline \multicolumn{7}{|c|}{$\begin{array}{l}\text { History of cancer other than cervical cancer including } \\
\text { familiar persons, } \%\end{array}$} \\
\hline Yes & 18.2 & 16.3 & 21.9 & 16.1 & 18.6 & $0.595^{\mathrm{d}}$ \\
\hline No & 81.5 & 83.3 & 77.7 & 83.5 & 81.4 & \\
\hline No answer & 0.3 & 0.4 & 0.4 & 0.4 & 0 & \\
\hline \multicolumn{7}{|c|}{$\begin{array}{l}\text { History of sexually transmitted disease including familiar } \\
\text { persons, \% }\end{array}$} \\
\hline Yes & 8.1 & 8.1 & 10.4 & 7.0 & 6.0 & $0.263^{d}$ \\
\hline No & 90.8 & 91.9 & 88.1 & 91.2 & 92.8 & \\
\hline No answer & 1.1 & 0 & 1.5 & 1.8 & 1.2 & \\
\hline
\end{tabular}

HPV, human papillomavirus; SD, standard deviation.

a One US dollar is roughly equivalent to 100 yen.

b ANOVA

${ }^{c}$ Chi-square test

${ }^{\mathrm{d}}$ Fisher's exact test 
Table 2. Changes in intention of vaccination before and four months after intervention across groups

\begin{tabular}{|c|c|c|c|c|c|c|c|c|c|c|}
\hline \multirow[b]{3}{*}{$\begin{array}{l}\text { Intention of vaccination, mean } \\
\text { (SD) }\end{array}$} & \multicolumn{3}{|c|}{$\begin{array}{l}\text { Statistics only } \\
\qquad(\mathrm{n}=258)\end{array}$} & \multicolumn{3}{|c|}{$\begin{array}{l}\text { Statistics and patient's } \\
\text { narrative } \\
(\mathrm{n}=269)\end{array}$} & \multicolumn{3}{|c|}{$\begin{array}{l}\text { Statistics and mother's } \\
\text { narrative } \\
(\mathrm{n}=284)\end{array}$} & \multirow[t]{3}{*}{$p^{\mathrm{b}}$} \\
\hline & Before & $\begin{array}{l}\text { Four } \\
\text { months } \\
\text { after }\end{array}$ & $p^{\mathrm{a}}$ & Before & $\begin{array}{l}\text { Four } \\
\text { months } \\
\text { after }\end{array}$ & $p^{\mathrm{a}}$ & Before & $\begin{array}{l}\text { Four } \\
\text { months } \\
\text { after }\end{array}$ & $p^{\mathrm{a}}$ & \\
\hline & $\begin{array}{c}2.58 \\
(0.94)\end{array}$ & $\begin{array}{c}2.62 \\
(1.00)\end{array}$ & $<0.256$ & $\begin{array}{l}2.53 \\
(0.94)\end{array}$ & $\begin{array}{c}2.60 \\
(0.97)\end{array}$ & $<0.054$ & $\begin{array}{c}2.47 \\
(0.95)\end{array}$ & $\begin{array}{c}2.54 \\
(0.95)\end{array}$ & $<0.096$ & \\
\hline $\begin{array}{l}\text { Changes in intention before and } \\
\text { four months after intervention, } \\
\text { mean (SD) }\end{array}$ & \multicolumn{3}{|c|}{$0.047(0.663)$} & \multicolumn{3}{|c|}{$0.077(0.651)$} & \multicolumn{3}{|c|}{$0.068(0.682)$} & 0.871 \\
\hline
\end{tabular}

$\mathrm{SD}=$ standard deviation

a $P$-values were assessed using the paired $t$-test.

${ }^{\mathrm{b}}$ A p-value was assessed using ANOVA.

\section{Figures}

\section{9}
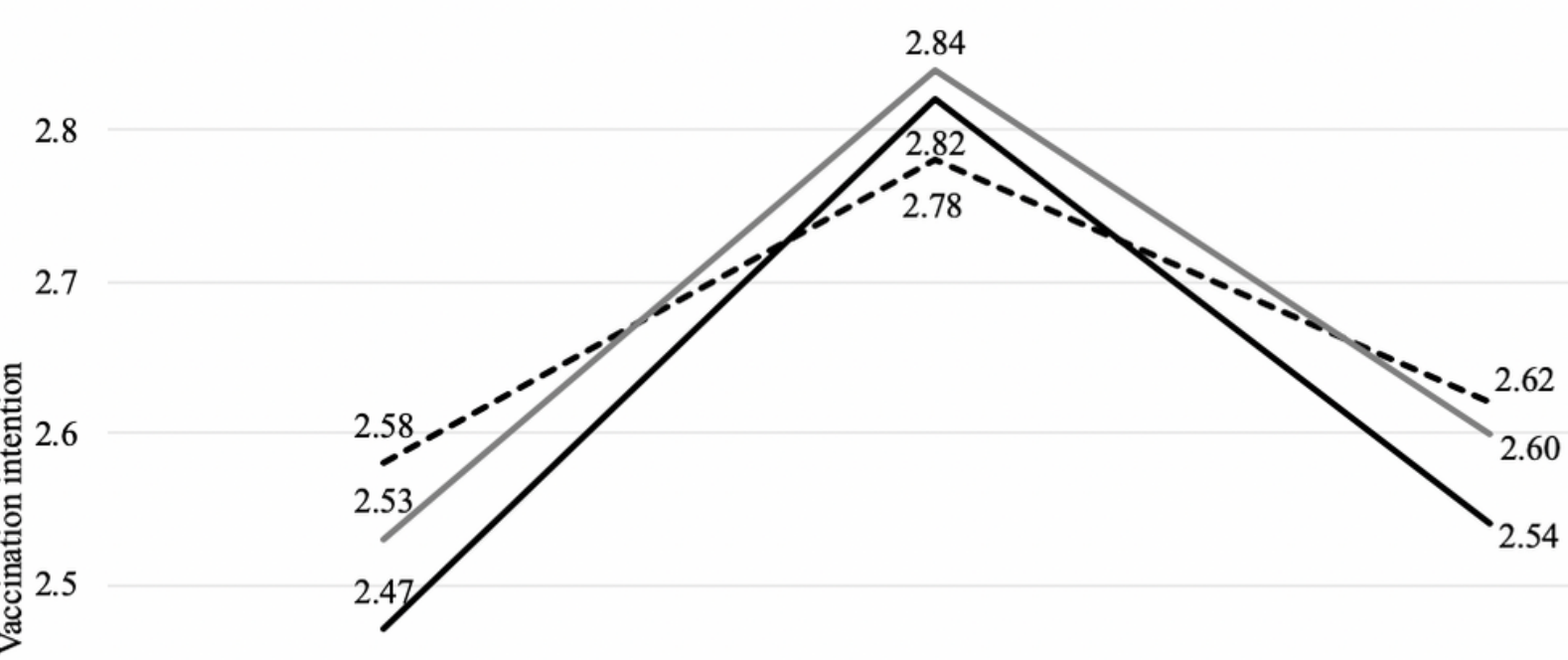

2.4

2.3

\subsection{8}

2.2

\section{Baseline}

- - Statistics only

- Statistics and mother's narrative
Directly after intervention

Four months after intervention

\section{- Statistics and patient's narrative \\ - - - Control (without intervention)}

\section{Figure 1}


Mean of vaccination intention at baseline, directly after, and four months after intervention across groups

\section{Supplementary Files}

This is a list of supplementary files associated with this preprint. Click to download.

- Appendix.docx 